\title{
WIE ANSÄTZE KÜNSTLICHER INTELLIGENZ DIE ÖFFENTLICHE VERWALTUNG UND DIE JUSTIZ VERÄNDERN KÖNNTEN
}

Jörn von Lucke und Jan Etscheid

Abstract: Künstliche Intelligenz (KI) bietet das Potenzial, Staat, Verwaltung, Justiz und Gesellschaft in hohem Maße disruptiv zu verändern. Bereits heute sind zahlreiche KI-Anwendungen Bestandteil von alltäglichen Produkten und Dienstleistungen. Doch wie können diese auch durch den öffentlichen Sektor systematisch genutzt werden? Welche Folgen sind damit für die Organisation von Staat und Verwaltung sowie die Gesellschaft verbunden? Zahlreiche offene Fragen bedürfen gesellschaftlicher Diskussionen rund um entscheidungsunterstützende Systeme und autonome Entscheidungen.

\section{Inhaltsverzeichnis}

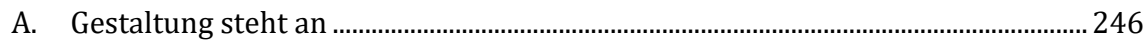

B. Sammelbegriff «Künstliche Intelligenz» ................................................................. 247

I. Definition und Abgrenzung ................................................................................. 247

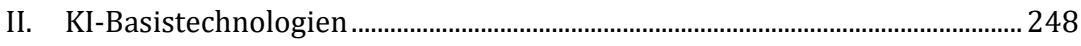

III. KI-Basisanwendungen ..................................................................................... 251

C. Anwendungssfelder für KI in der Verwaltung ....................................................... 252

I. Front-Office - Vordergrundverwaltung für den Bürgerkontakt.................... 253

II. Back-Office - Hintergrundverwaltung für die Sachbearbeitung.................... 255

III. Entscheidungsunterstützung ........................................................................... 256

IV. Entscheidungsautomatisierung: Entscheidende Systeme ............................... 258

V. Entscheidende Systeme mit Echtzeit-Entscheidungen .....................................2. 260

D. Anwendungsfelder für KI in der Justiz ..................................................................... 262

I. Entscheidungsunterstützende Systeme in der Justiz........................................ 262 
II. Entscheidende Systeme in der Justiz................................................................263

III. Mindestanforderungen .........................................................................................264

E. Neue Forschungsschwerpunkte ................................................................................266

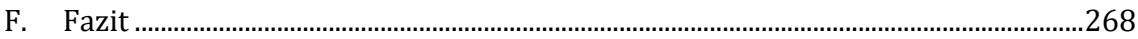

Literaturverzeichnis .............................................................................................................269

\section{A. GESTALTUNG STEHT AN}

Was ist eigentlich künstliche Intelligenz? Wie werden künstliche Intelligenzen unser zukünftiges Leben beeinflussen? Obwohl der Begriff der «künstlichen Intelligenz» (KI) bereits seit über 50 Jahren verwendet wird, gewinnen diese Fragen durch den aktuellen technischen Fortschritt enorm an Relevanz. Auf der einen Seite werden dunkle Visionen von Maschinen gezeichnet, die die Menschheit beherrschen. Andere Visionäre sehen KI als ein Wundermittel zur Lösung aller Probleme der Welt auf einfachste Weise. Doch was steckt wirklich hinter KI? Und wozu lässt sie sich einsetzen?

Ein intelligent vernetztes Regierungs- und Verwaltungshandeln (Smart Government) wird die Möglichkeiten smarter Objekte und cyberphysischer Systeme (CPS) zur effizienten wie effektiven Erfüllung öffentlicher Aufgaben nutzen. Mit dem Schlagwort «Smart Government» wird die Anwendung des Internets der Dinge und des Internets der Dienste im Rahmen der Prozesse des Regierens und Verwaltens konkretisiert. Dabei geht es um weit mehr als nur um die technische Integration von smarten Objekten und CPS in die öffentliche Verwaltung. ${ }^{1}$ Gerade in Verbindung mit künstlicher Intelligenz bietet sich darüber hinaus die Möglichkeit, in völlig neue Dimensionen der Unterstützung und Automatisierung von Prozessen vorzustoßen.

Es mag zahlreiche Gründe wie etwa Geschwindigkeit, Wirtschaftlichkeit und Kontrolle geben, die für den Einsatz autonomer Systeme im öffentlichen Sektor sprechen. Ebenso gibt es zahlreiche Argumente, die aus Sorge vor einer solchen Entwicklung formuliert werden, um den Menschen ihre Entscheidungsspielräume zu erhalten. Technisch lassen sich autonome Systeme zunehmend leichter konzipieren, implementieren und in Betrieb nehmen. Aber sind Staat, Verwaltung und Justiz schon darauf vorbereitet? 
Welche Herausforderungen müssen benannt und zufriedenstellend beantwortet werden, bevor man autonome Systeme guten Gewissens für die Erledigung öffentlicher Aufgaben dauerhaft einsetzen sollte?

Dieser Beitrag wird aufzeigen, welche Fähigkeiten und Einsatzpotenziale hinter dem Begriff der «künstlichen Intelligenz» liegen. Aufbauend auf einer Definition des Begriffs und den damit verbundenen Basistechnologien und Basisanwendungen werden fünf Anwendungsfelder für die öffentliche Verwaltung skiziert, in denen KI eingesetzt werden kann. Anschließend werden einige Anwendungsfelder für KI in der Justiz aufgezeigt. Dies resultiert in der Herausarbeitung von Grenzen für den Einsatz von KI in Staat und Justiz sowie der Bestimmung neuer Forschungsschwerpunkte.

\section{B. SAMMELBEGRIFF «KÜNSTLICHE INTELLIGENZ»}

\section{Definition und Abgrenzung}

Im Kontext der Digitalisierung ist die «künstliche Intelligenz» (KI) zu einem der prägenden Begriffe geworden. Viele Unternehmen und Organisationen beschäftigen sich derzeit mit den sich daraus ergebenden Möglichkeiten. Sie verwenden den Begriff und die damit zusammenhängenden Technologien aber in ganz unterschiedlichen Kontexten. Zugleich gewinnt die Thematik in Presseartikeln, Vorträgen und Strategiepapieren an Bedeutung. Nicht mehr nur die Experten und Informatiker am Deutschen Forschungszentrum für Künstliche Intelligenz (DFKI), sondern Wissenschaftler aus unterschiedlichsten Disziplinen befassen sich mit künstlicher Intelligenz und den damit einhergehenden technischen, gesellschaftlichen oder organisationalen Veränderungen. Dabei bleibt häufig unklar, was jedoch eigentlich hinter dem Begriff der künstlichen Intelligenz steht. Dabei dominiert die Erwartung, dass bislang zwingend von Menschen ausgeführte Vorgänge nun auf technische Systeme vollständig übertragen werden können und dass diese Systeme dabei selbständig lernen, ihre Aufgaben immer besser zu erledigen.

Zum heutigen Zeitpunkt existiert keine allgemein anerkannte Definition künstlicher Intelligenz. Vielmehr handelt es sich um einen Sammelbegriff unterschiedlicher 
Technologien und Ansätze in verschiedenen Reifegraden mit unterschiedlichen Sicherheits- und Vertrauensniveaus. Der Begriff der künstlichen Intelligenz legt nahe, dass es sich dabei um Systeme handelt, welche menschliche Intelligenz nachbilden sollen. Die KI als Subdisziplin der Informatik nutzt unterschiedliche Technologien und Architekturen. ${ }^{2}$ Deshalb wird KI auch als Querschnittstechnologie bezeichnet. ${ }^{3}$ Dementsprechend erscheint die Verwendung eher breit angelegter Definitionen von KI zielführender zu sein, welche stärker auf den Output als auf die Systeme selbst ausgerichtet sind. Eine derartige Arbeitsdefinition des deutschen KI-Forschers Klaus Mainzer beschreibt KI als Systeme, die «selbstständig effizient Probleme lösen können».4 Marvin Minsky definierte bereits $1966 \mathrm{KI}$ etwa als die Wissenschaft, Maschinen Dinge machen zu lassen, die Intelligenz erfordern würden, wenn dies Menschen tun würden.

Künstliche Intelligenz ausschließlich von der technologischen Seite her zu definieren erscheint über den bisherigen zeitlichen Verlauf hinweg nur schwer möglich zu sein. Vor 50 Jahren standen gänzlich andere technische Möglichkeiten im Mittelpunkt als heute. Zwischenzeitlich hat sich in der Wissenschaft die Unterscheidung zwischen schwacher KI, starker KI und einer Superintelligenz durchgesetzt. Schwache KI wird in der Regel für bestimmte Anwendungen entwickelt und genutzt. Konkret handelt es sich dabei zum Beispiel um Expertensysteme, Spracherkennung, Navigations- oder Übersetzungsdienste. Auf schwacher KI basierende Anwendungen sind heute bereits vielfach im Einsatz und finden sich etwa in Form von intelligenten Suchvorschlägen oder optimierten Routenberechnungen im alltäglichen Leben. ${ }^{5}$

\section{KI-Basistechnologien}

Aufgrund der schwierigen technologischen Abgrenzung erscheint es zielführender KI anhand ihrer Fähigkeiten zu differenzieren. Diese sollen im Nachfolgenden als KI-

2 Fachbereich Künstliche Intelligenz (FBKI) der Gesellschaft für Informatik e.V.: https://fb-ki. gi.de.

Vgl. DJEFFAL 2017.

Vgl. MAINZER 2016.

Vgl. ETSCHEID/VON LUCKE 2019. 
Basistechnologien beschrieben werden, da sie Grundfähigkeiten bereitstellen. Gleichzeitig darf jedoch auch nicht unerwähnt bleiben, dass sich KI durchaus auch von der technischen Seite beschreiben lässt. So lassen sich KI-Ansätze beispielsweise anhand der Kategorien verwendeter Lernmethoden, Systemarchitekturen sowie Algorithmen unterscheiden. KI-Lernmethoden umfassen dabei unter anderem das tiefe Lernen (Deep Learning), überwachtes Lernen, unüberwachtes Lernen, bestärkendes Lernen sowie Meta-Lernen. Systeme und Architekturen lassen sich etwa in intelligente Agenten, Expertensysteme, Entscheidungsunterstützungssysteme, RegelmanagementSysteme, künstliche Immunsysteme und Quantenlogik-Systeme einteilen. Die Technologien, auf welchen diese Systeme basieren, sind ebenfalls sehr vielfältig. Neben künstlichen neuronalen Netzen kann dazu auch auf Support Vector Machines, possibilitische Netzwerke, Multi-Kontext-Systeme oder genetische Algorithmen gesetzt werden. ${ }^{6}$

Für die konkrete Anwendung ist die zugrundeliegende Technologie oft zweitrangig. Vielmehr stellen sich die Fragen, zu welchem Zweck KI-Basistechnologien eingesetzt werden können, was sie zu leisten im Stande sind und welche Grenzen gesetzt werden sollten. Die wichtigsten KI-Basistechnologien werden im Folgenden ${ }^{7}$ kurz vorgestellt.

KI-basierte Mustererkennung analysiert Daten, um darin Regelmäßigkeiten, Wiederholungen, Ähnlichkeiten oder Gesetzmäßigkeiten zu erkennen. IT-Systeme sind vielfach in der Lage, wesentlich größere Datenmengen in kürzerer Zeit zu analysieren, als dies einem Menschen jemals möglich wäre. Zudem treten bei IT-Systemen auch keine Ermüdungserscheinungen oder Flüchtigkeitsfehler auf. Ein Vorteil lernender Systeme der Mustererkennung ist die Identifizierung von Zusammenhängen, welche dem Menschen zuvor weder bekannt noch aufgefallen waren. Daten können nicht nur auf zuvor definierte Zusammenhänge überprüft, sondern auch ergebnissoffen ausgewertet werden. Anwendungsfälle hierfür finden sich bereits bei der Abwehr von Cyberangriffen, in der Medizin ${ }^{8}$ sowie der Verknüpfung von Wissensgebieten. ${ }^{9}$

6 Vgl. ausführliche Darstellung im ÖFIT-Trendsonar: WENZEL/GROSCH 2018.

7 Vgl. ETSCHEID/VON LUCKE 2019.

8 Vgl. KÖNEKE 2018.

9 Vgl. LINDINGER 2019. 
KI-basierte Texterkennung nutzt Algorithmen, um Informationen aus unstrukturierten Daten sowie Inhalte aus natürlicher Sprache in maschinenlesbare und damit weiterzuverarbeitende Form zu transformieren. KI-basierte Algorithmen sind dabei nicht nur in der Lage, Wörtern eine Bedeutung zuzuordnen, sondern diese auch im Zusammenhang mit weiteren Wörtern zu bewerten. Unstrukturierte Daten werden damit auch für technische Systeme verarbeitbar.

KI-basierte Systeme können auch in die Lage versetzt werden, akustische Signale und Tonfolgen zu erkennen und diese bestimmten Ereignissen oder Verursachern zuzuordnen. Dabei kann es sich um die Erkennung von Ereignissen, wie dem Vorbeifahren eines Zuges anhand von natürlichen Umgebungsgeräuschen, aber auch um die Betriebsgeräusche eines Motors handeln, anhand denen sich ein anbahnender Defekt frühzeitig erkennen lässt.

Darauf aufbauend kombiniert die KI-basierte Spracherkennung die Fähigkeiten aus Texterkennung und akustischer Erkennung. Algorithmen ermöglichen es, gesprochene Sprache zu verschriftlichen und in maschinenlesbare Form zu übersetzen. Hierzu werden die Informationen aus der akustischen Aufnahme extrahiert und in strukturierte Form überführt.

KI-basierte Übersetzungsdienste ermöglichen die Übersetzung von in natürlicher Sprache vorliegender Texte in andere Sprachen. Fremdsprachen, leichte Sprache oder Zeichensprache lassen sich so in eine Amtssprache übersetzen. Aber auch umgekehrt ist dies möglich. Hierfür läuft in einem ersten Schritt zunächst eine Spracherkennung ab. Die dabei gewonnenen Daten werden in einem zweiten Schritt in die gewünschte Sprache übersetzt und dargestellt oder vorgelesen.

KI-basierte Bilderkennung ist in der Lage, Objekte in Bildern zu identifizieren und diese Kategorien zuzuordnen. Da Gegenstände wie ein Auto nicht immer gleich aussehen oder aus unterschiedlichen Positionen aufgenommen werden können, muss das System in der Lage sein, situativ bestimmte Merkmale erkennen zu können.

Die Gesichtserkennung als Sonderfall der Bilderkennung erkennt menschliche Gesichter auf Basis eindeutiger biometrischer Merkmale. Damit können Personen anhand geometrischer Strukturen ihres Gesichts identifiziert oder Emotionen analysiert werden. 
Die 3D-Raumerkennung stellt wiederum eine weitere Stufe der Bilderkennung dar. Bilder werden nicht mehr nur zweidimensional analysiert. Mindestens zwei Bilder sind erforderlich, um zu einem dreidimensionalen Bild kombiniert zu werden, was räumliche Analysen ermöglicht. So wohl über Bilderkennung als auch weitere Sensoren können Entfernungen und Positionen im dreidimensionalen Raum erkannt und verarbeitet werden.

Die KI-basierte Gesten- und Bewegungsmustererkennung setzt auf die Analyse von Bewegungsdaten (Videofilme, Bewegungssequenzen) einer Person. Menschliche Gesten und Bewegungen sind ebenfalls eindeutige biometrische Merkmale, an Hand derer Personen identifiziert werden können.

\section{KI-Basisanwendungen}

Aufbauend auf diesen Basistechnologien kann KI zur Simulation unterschiedlicher menschlicher Fähigkeiten genutzt werden. ${ }^{10}$ Die bedeutendsten Basisanwendungen sollen kurz dargestellt werden:

KI-basierte Wahrnehmung bezeichnet die Analyse von Daten zur Erkennung von Umweltveränderungen, Einstellungen und Emotionen. Hierzu verarbeitet das System generierte (Sensor-)Daten und ordnet diese Kategorien zu, sodass einzelne Daten zu Ereignissen oder emotionalen Einstellungen aggregiert werden können.

Bei der KI-basierten Benachrichtigung steht die Reaktion des Systems im Vordergrund. Erkannte Muster, Ereignisse oder Emotionen werden dazu genutzt, um Anwender gezielt zu benachrichtigen. Anwender werden so auf Ereignisse oder Zustände hingewiesen, damit sie darauf zeitnah und angemessen reagieren können.

KI-basierte Empfehlungen erweitern die Datenauswertung dahingehend, dass ausgehend davon nicht nur der Status Quo dargestellt wird, sondern auch Handlungsempfehlungen für den Anwender gegeben werden. Hierzu ist es nicht nur notwendig, den Daten Kategorien zuzuordnen. Zudem muss der wahrgenommene Ist-Zustand mit ei- 
nem Soll-Zustand abgeglichen werden, um anhand der festgestellten Abweichung Empfehlungen zum Erreichen des Soll-Zustands geben zu können.

KI kann auch zur Erstellung von Vorhersagen und Prognosen eingesetzt werden. Ausgehend von den in den Daten erkannten Mustern werden Vorhersagen zur weiteren Entwicklung abgeleitet, welche dem Anwender angemessen mitgeteilt werden.

KI-basierte Vorsorge verknüpft die Prognose mit dem Abgleich von Soll-/Ist-Zustand, sodass prognostizierte Abweichungen erkannt und frühzeitig Empfehlungen oder Warnhinweise mit der Bitte um Behebung gegeben werden können.

Neben den bisherigen entscheidungsunterstützenden Fähigkeiten kann KI auch zum Treffen selbstständiger Entscheidungen eingesetzt werden. Hierbei wird der Entscheider nicht durch Datenauswertungen oder Prognosen in seinem Entscheidungsprozess unterstützt. Vielmehr reagiert das System auf die Daten mit eigenständig getroffenen bindenden Entscheidungen, sodass der Anwender (und damit der Mensch) völlig aus dem Vorgang herausgenommen wird.

Dabei kann die Datenauswertung im Rahmen von KI-basierter Situationswahrnehmung auch in Echtzeit erfolgen. Hierbei müssen die oben dargestellten Fähigkeiten innerhalb von Millisekunden ausgeführt werden. Solche Systeme können somit nahezu in Echtzeit eine Situation bewerten und mit Hinweisen, Alarmen, Prognosen oder Entscheidungen sofort reagieren.

\section{ANWENDUNGSSFELDER FÜR KI IN DER VERWALTUNG}

Künstliche Intelligenz kann für unterschiedliche Aufgabentypen eingesetzt werden. Die KI-Basistechnologien und -Anwendungen haben aufgezeigt, welche menschlichen Fähigkeiten zur Erfüllung von Aufgaben bereits heute durch KI übernommen werden können. An fünf Anwendungsfelder sollen exemplarisch aufzeigt werden, wie KI als weiterer Modernisierungstreiber in der Verwaltung genutzt werden kann. 


\section{Front-Office - Vordergrundverwaltung für den Bürgerkontakt}

Eine stärkere Ausrichtung des Verwaltungszugangs an den Bedürfnissen der Bürger ist kein neues Phänomen. Die aus der Privatwirtschaft bekannte Kundenorientierung wird bereits seit Jahrzehnten auch im Kontext der öffentlichen Verwaltung als Bürgerorientierung immer stärker eingefordert.11 In den Augen vieler Bürger liegt die Verwaltung in dieser Hinsicht noch weit hinter der Privatwirtschaft zurück. Dabei bieten sich durch künstliche Intelligenz derzeit neuartige Möglichkeiten, um dem Bürger den Kontakt mit der Verwaltung so einfach und angenehm wie möglich zu gestalten.

Eines der bekanntesten Beispiele für den Einsatz von künstlicher Intelligenz sind Chatbots, also Dialogsysteme, mit denen über natürliche Sprache text- oder sprachbasiert kommuniziert werden kann. Chatbots können dabei bis zu einem gewissen Maß den Dialog mit einem Menschen simulieren. ${ }^{12}$ Nutzer können ihre Anfragen in natürlicher Sprache stellen, als ob sie mit einem Menschen sprechen würden. Sie bekommen zeitnah auf die gleiche Weise eine Antwort ausgegeben. Chatbots können damit die bestehende Zugangskanäle zur Verwaltung erweitern. Sie ermöglichen den Nutzern, dass sie ohne Fachkenntnisse die gewünschten Informationen finden und diese in für Laien verständlicher Form erhalten. Beispielsweise mit einem Bürgerkonto verknüpfte, personalisierte Chatbots können Nutzereingaben auch mit zum Bürger vorhandenen Daten verknüpfen und so die Qualität der Antworten verbessern. Auch können durch KI Emotionen erkannt werden, so dass angemessen reagiert werden kann. ${ }^{13}$ Noch sind Chatbots zumeist Informationsinstrumente, die über Leistungen, Abläufe und Fristen informieren. Künftig werden über Chatbots auch Anträge gestellt und sogar Prozesse vollständig durchgeführt werden können. Die in Antragsverfahren notwendigen Informationen würden dabei in natürlicher Sprache erfasst und durch den Chatbot zur weiteren

11 Vgl. Duncker/Noltemeier 1985, Grunow 1988, Daum 2002 und Möltgen/Lorig 2009.

12 Vgl. STUCKI/D'OnOFRIO/PORTMANn 2018.

13 Vgl. KuHn 2019. 
Verarbeitung in eine strukturierte Form übersetzt und dem Fachverfahren übermittelt.

Persönliche Sprachassistenten unterscheiden sich gegenüber Chatbots dadurch, dass sie auf eine hörbare Kommunikation in natürlicher Sprache und einer Steuerung ausschließlich über Sprache setzen. Sie können etwa in ein Smartphone, einen Rechner oder einen smarten Lautsprecher integriert sein und werden über ein Aktivierungswort gestartet. Plattformbasierte Sprachassistenten nutzen zahlreiche KI-basierten Dienste im Hintergrund. Erste Städte in Deutschland erproben bereits die Nutzung von KI-basierten Sprachassistenzsystemen. ${ }^{14}$

Im Verwaltungskontext tragen Portale einem einheitlichen Zugang Rechnung. ${ }^{15}$ Noch stärkere Anreize könnte ein Ausbau der Bürgerkonten hin zu einem persönlichen Assistenten für unterschiedlichste Lebensbereiche setzen. Der Nutzer erhält dabei die einstellbare Möglichkeit, seine bei der Verwaltung vorliegenden Daten auch für Dienstangebote Dritter freizugeben. Unternehmen würden von der hohen Qualität und dem hohen Vertrauen in die Verwaltungsdaten profitieren. Ein Zugriff auf Identitäten und aktuelle Meldeanschrift ließe etwa den Aufwand zur Identifikation für viele Dienste erheblich sinken. Gleichzeitig könnte durch eine intelligente Verknüpfung und Auswertung beispielsweise ein Reiseanbieter im Rahmen der Buchung einer Auslandsreise auf das Ablaufen des notwendigen Ausweisdokuments hinweisen. Nicht entstehen darf jedoch der Eindruck eines Assistenten, der gläserne Bürger schafft. Die Bürger entscheiden selbst, wer Zugriff auf ihre hinterlegten Daten haben darf. Standardmäßig wäre dies niemand (Privacy-by-Design). Technisch darf eine Verknüpfung gegen den Willen der Bürger gar nicht erst möglich sein.

Die meisten Verwaltungskontakte dienen der Beantragung von Verwaltungsleistungen. ${ }^{16}$ Schritte dahin sollten für die Bürger so weit wie möglich vereinfacht werden. Tatsächlich basieren viele Antragsverfahren noch immer auf analogen, formularbasierten Vordrucken und Verfahren. Erste Herausforderung für die Antragsteller ist das Auf-

Vgl. StanoevsKa-SlabeVA 2018, S. 27 ff. und AREeKadan 2018, S. 22.

Vgl. vON LUCKE 2008.

Vgl. GOLDACKER 2017. 
finden des notwendigen Formulars. Neben dem Auffinden ermöglicht KI auch eine vereinfachte Antragstellung, etwa indem die Daten aus der natürlichen Sprache direkt übernommen, auf Plausibilität oder Richtigkeit geprüft und in ein elektronisches Formular eingetragen werden. Im idealen Fall können Bürger ihr Anliegen in natürlicher Sprache schriftlich oder mündlich vorbringen. Anders ausgedrückt benötigt der Bürger somit weder die Kenntnisse über das passende Verfahren noch müssen seine Angaben von ihm selbst in ein papierbasiertes Formular überführt werden.

\section{Back-Office - Hintergrundverwaltung für die Sachbearbeitung}

Mit steigender Größe und Komplexität einer Organisation steigt neben der eigentlichen Leistungserstellung der Bedarf an Unterstützungsprozessen. Diese Prozesse generieren selbst keine Wertschöpfung, ermöglichen jedoch erst die eigentlich gewünschten Prozesse. ${ }^{17}$ In einer derart thematisch breit aufgestellten und komplexen Organisation wie der öffentlichen Verwaltung nehmen Unterstützungsprozesse einen nicht unerheblichen Anteil ein.

Zur Vollauslastung der Mitarbeiter muss bekannt sein, über welche Fähigkeiten ein Mitarbeiter verfügt sowie welche freien Kapazitäten individuell sowie in der Behörde vorhanden sind. Ein intelligentes System kann nicht nur die Eignung eines Mitarbeiters für einen speziellen Fall anhand von zugewiesenen Fähigkeiten und Erfahrungen beurteilen, sondern auch die organisationale wie individuelle Auslastung erkennen, um auf Engpässe und Lufträume frühzeitig und angemessen zu reagieren. Ebenso können Teilprozesse priorisiert werden, weil beispielsweise ein wichtiger Gesamtprozess ansonsten verzögert würde.

Ansatzpunkte bietet auch die klassische Poststelle in Verbindung mit der elektronischen Poststelle. Sie nehmen postalische Sendungen, Aktenpakete, Faxe und zunehmend auch Emails entgegen. Diese Schriftstücke beziehungsweise die darin enthalten Informationen müssen anschließend an die richtige Stelle gelangen. Mit Einscannen 
und KI-basierter Texterkennung lassen sich die Inhalte der Schreiben rasch auszuwerten, so dass sie elektronisch über Vorgangsbearbeitungssysteme an die richtige Abteilung und den zuständigen Bearbeiter weitergeleitet werden können.

Interne Vorgänge wie die Abrechnung von Reisekosten lassen sich mit Hilfe künstlicher Intelligenz stark vereinfachen. KI-basierte Risikomanagementsysteme können eine Vorauswahl treffen, nur noch fragwürdige Vorgänge genauer zu betrachten. Alle unkritischen Vorgänge werden durch das System direkt freigegeben. ${ }^{18}$

Daneben existieren zahlreiche weitere Unterstützungsprozesse mit einem hohen Potenzial für Vereinfachung oder Automatisierung. $\mathrm{Zu}$ nennen sind hier unter anderem der IT-Helpdesk für Mitarbeiter, die Übersetzung von Dokumenten oder die Anfertigung von Gesprächsprotokollen. Obwohl viele Prozesse durch die Integration von Softwareprodukten bereits vereinfacht wurden, sind in diesen Prozessen noch immer Eingriffe der menschlichen Bearbeiter notwendig. Das Zusammenführen von Daten aus unterschiedlichen Quellen, der Umgang mit unerwarteten Informationen oder das Treffen von Entscheidungen werden häufig durch den Menschen ausgeführt. Robotic Process Automation (RPA) setzt an der Bedienung der bestehenden und sich vielfach über lange Zeiträume bewährten Softwareprodukte an. Lediglich wird die Bedienung dieser Software nicht mehr durch einen Menschen, sondern durch automatisierte Algorithmen vorgenommen. ${ }^{19}$ Bildlich gesprochen bedient ein Roboter virtuell die Tastatur und Maus des Computers und ermöglichen damit die Automatisierung bestehender IT-Anwendungen.

\section{Entscheidungsunterstützung}

In der öffentlichen Verwaltung wird eine Vielzahl an unterschiedlichen Entscheidungen mit rechtlicher Bindung getroffen. Die eigentliche Entscheidung setzt sich stets aus der Abwägung möglicher und der endgültigen Festlegung auf eine Alternative zusammen. Da die Mehrzahl der Verwaltungsverfahren Entscheidungen beinhalten, wird die

Vgl. LANZ 2010.

19

Vgl. SCHEER 2017. 
Verwaltungswissenschaft zuweilen auch als «Entscheidungswissenschaft» bezeichnet. ${ }^{20} \mathrm{Im}$ Kontext einer evidenzbasierten Regierungslehre (Evidence Based Government), idealtypisch nahezu in Echtzeit, gewinnen Entscheidungen auf Basis von Daten und Fakten derzeit stark an Bedeutung.

Die öffentliche Verwaltung besitzt bereits riesige Mengen an Daten und Informationen aus unterschiedlichen Quellen, die im Rahmen der Wahrnehmung öffentlicher Aufgaben erhoben werden müssen. Zusätzlich sind smarte Objekte aus dem Alltag der Bürger heute nicht mehr wegzudenken. Smartphones, smarte Uhren, smarte Messstellen (Smart Meter) oder smarte Hauselektronik (Smart Home) generieren laufend riesige Datenmengen, an denen staatliche Stellen zunehmend ein hohes Interesse haben. ${ }^{21}$ Die Verwaltung selbst erhebt ebenfalls smarte Daten, etwa über Sensorik oder Verkehrsleitsysteme. Oftmals werden die Potenziale dieser Daten jedoch nur teilweise genutzt. Die Aufbereitung über sogenannte Dashboards oder Cockpits stellt einen ersten Schritt zur Nutzung dar. Das System erkennt dabei, welche Daten in diesem Fall relevant sind, berechnet daraus Kennzahlen, visualisiert zentrale Argumente oder stellt Bezüge zu früheren Entscheidungen her. Der Bearbeiter erhält auf diese Weise eine fundiertere Entscheidungsgrundlage, auf welcher er seine persönliche Einschätzung treffen kann.

Eine wichtige Form der Entscheidungsunterstützung im Verwaltungsalltag ist die intelligente Einsatzplanung. Ein bekanntes Beispiel für KI-basierte Einsatzplanung ist die vorausschauende Polizeiarbeit (Predictive Policing). ${ }^{22}$ Doch auch in anderen Bereichen können solche Risikomanagementsysteme eingesetzt werden. Denkbar sind beispielhaft auch vorrausschauende Wartungsarbeiten, indem Sensoren ungewöhnliche Töne aus den Maschinen registrieren und auf eine erforderliche Wartung aufmerksam machen, bevor die Maschine ausfällt. Aber auch ohne konkrete Sensordaten können aufgrund statistischer Auswertung Wartungsbedarfe prognostiziert oder der Personalbedarf für eine Einsatzschicht berechnet werden. Ziel dieser Ansätze ist es, die vorhandenen Ressourcen effizienter einzusetzen.

Vgl. PÜtTnER 2000, S. 332 und NeSSELDREHER 2006.

21

Vgl. vON LUCKE 2018a.

22

Vgl. KNOBLOCH 2018. 
Unterstützt werden können die Sachbearbeiter, Entscheider, verantwortlichen Vorgesetzten und die überprüfende Revision auch durch eine Prüfung und zweite Einschätzung des Outputs. Ein Entscheidungskontrollradar kann den Entscheider dahingehend unterstützen, dass die Entscheidung ganz im Sinne des Vier-Augen-Prinzips vor dem Vollzug nochmals geprüft wird. Weicht die Entscheidung auf Basis der vorhandenen Daten von der vom System erwarteten Entscheidung ab, so wird dem Bearbeiter zunächst direkt eine Meldung mit Überarbeitungsempfehlung ausgegeben.

Auch der Entscheidungsvorgang selbst kann durch technische Systeme unterstützt werden. Im Entscheidungsprozess können dem Bearbeiter somit ein oder mehrere Vorschläge vorgelegt werden, gegebenenfalls mit Kennzahlen versehen, welche dieser prüft und anschließend bei Richtigkeit und Passung annehmen, gegebenenfalls anpassen oder verwerfen kann. Auf diese Weise können Bearbeiter Routinefälle wesentlich schneller bearbeiten, in Sonderfällen aber auch problemlos von den gemachten Vorschlägen abweichen. Die Entscheidungsmacht bleibt bei entscheidungsunterstützenden Systemen stets beim Menschen, vom Sachbearbeiter bis zum Entscheider und Politiker. Gleichzeitig kann auch die langfristige Wirkung von Entscheidungen stärker berücksichtigt werden. Prognosen zu den Langzeitfolgen einer Entscheidung lassen sich durchrechnen und anhand von Kennzahlen oder Visualisierungen verständlich aufzeigen. Diese können wertvolle Erkenntnisse liefern und eine stärkere Nachhaltigkeit der zu treffenden Entscheidung sichern.

\section{Entscheidungsautomatisierung: Entscheidende Systeme}

Neben der Unterstützung des Entscheidungsträgers kann KI auch zur Automatisierung von Entscheidungen eingesetzt werden. Dies bedeutet, dass der Mensch aus dem Entscheidungsprozess herausgenommen wird und die verbindlichen Entscheidungen autonom und damit ausschließlich durch ein technisches System getroffen werden.

Die Ergebnisse zahlreicher Verwaltungsentscheidungen lassen sich direkt aus den einschlägigen Gesetzestexten ableiten. Hierfür ist weder eine Interpretation des Sachverhalts noch der daraus resultierenden Folgen notwendig. Es muss lediglich geprüft werden, inwiefern die kraft Gesetzes definierten Voraussetzungen erfüllt sind, sodass die direkte Folge abgeleitet werden kann. In diesen Fällen sind Verwaltungsmitarbeiter 
häufig damit beschäftigt, die Voraussetzungen für bestimmte Verfahren oder Leistungen anhand klar messbarer Fakten und Kriterien zu prüfen. Nach diesem Muster ließen sich zahlreiche Verfahren vollautomatisiert abwickeln, ohne dass ein Verwaltungsmitarbeiter in die Bearbeitung mit einbezogen werden müsste. Liegen die erforderlichen Informationen in geeigneter Form vor und sind die notwendigen Schnittstellen vorhanden, ist die technische Umsetzung meist keine große Herausforderung. Derzeit sind es vor allem rechtliche Hürden, welche die Vollautomatisierung noch erschweren. ${ }^{23}$ Noch immer geht das deutsche Rechtssystem davon aus, dass eine Entscheidung letztlich einer Person zuzuordnen ist und diese auch dafür in Haftung genommen werden kann.

Daneben existieren in der Verwaltung auch Entscheidungen, deren Ergebnisse sich nicht direkt aus den gesetzlichen Vorgaben ableiten lassen, sondern die im Einzelfall abgewogen werden müssen. Diese werden als Entscheidungen mit Spielräumen für Beurteilung und Ermessen bezeichnet. Der Gesetzgeber in Deutschland hat diese Entscheidungen explizit in §35a VwVfG von der Vollautomatisierung ausgeschlossen. ${ }^{24}$ Die Herausforderung in den kommenden Jahren besteht dabei vor allem in der Erkennung von vorhandenen Spielräumen und ihrer ordnungsgemäßen Nutzung. Spielräume werden vom Gesetzgeber vor allem mit der Intention eingeräumt, im Einzelfall die intendierte Wirkung herzustellen, auch wenn der Einzelfall im Vorfeld nicht genau absehbar war. Insofern ist auch der gesamte Entscheidungsprozess deutlich komplexer. Es muss ein Verständnis dafür vorhanden sein, welche Wirkung man erreichen möchte und welche Mittel dazu im Einzelfall anzuwenden sind. Insofern besteht die Entscheidung nicht nur aus einer Übertragung der gesetzlichen Vorgaben auf den Einzelfall und die abgeleiteten Folgen, sondern es muss eine Einordnung und Abwägung mehrerer Faktoren vorgenommen werden. Obwohl die Komplexität von Ermessensentscheidung deutlich höher einzuschätzen ist, erscheint es nicht sinnvoll, dies als absolutes Kriterium für eine Automatisierbarkeit heranzuziehen. Die erste Herausforderung besteht darin, vorhandenes Ermessen zu erkennen. Die zweite Herausforderung für ler-

Vgl. SIEGEL 2017.

24 Vgl. BRAUn Binder 2016. 
nende Entscheidungssysteme liegt darin, den im jeweiligen Fall vorliegenden Ermessensspielraum zu erfassen und innerhalb dieses Raumes eine angemessene Entscheidung zu treffen.

\section{Entscheidende Systeme mit Echtzeit-Entscheidungen}

Neben Verwaltungsprozessen, bei denen Entscheidung und Abwicklung innerhalb von Minuten, Stunden oder Tagen problemlos realisierbar ist, erfordern andere Verwaltungsentscheidungen eine Entscheidungsfindung und Umsetzung nahezu in Echtzeit. Dies hängt einerseits mit der Notwendigkeit einer direkten Reaktion zusammen, etwa einer Reaktion im Straßenverkehr im Falle von Ampelsteuerungen oder autonom fahrenden Fahrzeugen. Andererseits kann auch die Wirkung von Prozessen stark verbessert werden. Vielfach wird es dadurch möglich, statt auf die sichtbaren Folgen erst in hohem zeitlichem Abstand, sofort auf die Ursachen zu reagieren und so Eigentum und Menschenleben zu schützen.

Intelligent vernetzte und KI-basierte Verkehrsleitsysteme bieten die Möglichkeit, nahezu in Echtzeit auf Verkehrslagen zu reagieren. Bereits heute werden zahlreiche verkehrsbezogene Daten erhoben. Fahrzeuge erfassen ständig vielfältige Daten, auch zu ihrem Standort, Geschwindigkeiten und Energieverbrauch. Fußgänger und Radfahrer sind anhand ihrer Smartphones, Bluetooth- oder GPS-Tracker lokalisierbar und verfolgbar. Weitere externe Datenquellen lassen sich einbinden. Ist beispielsweise bekannt, dass eine Großveranstaltung zu einem bestimmten Zeitpunkt endet, können die erforderlichen ÖPNV- und Straßenkapazitäten hierzu bereits frühzeitig gesichert und bereitgestellt werden. Zu diesem Zweck benötigt man urbane Mobilitätsdatenräume, in denen die verschiedenen Daten gesammelt werden. Auf dieser Basis können KI-basierte Entscheidungen über Ampelschaltungen, Umleitungen oder Tempolimits nahezu in Echtzeit getroffen werden. Auch intelligente grüne Wellen für Rettungs- und Einsatzfahrzeuge oder Busse und Straßenbahnen können durch Algorithmen so gesteuert werden, dass die Straßen möglichst von Fahrzeugen frei bleiben und keine Kreuzungen verstopfen.

Katastrophenmanagement erfordert im Regelfall eine schnellstmögliche Reaktion. Im Falle von Naturkatastrophen oder Terroranschlägen kann eine zeitnahe Reaktion 
dazu beitragen, Menschenleben zu retten. Oftmals vergeht durch Abstimmung und Kommunikation jedoch wertvolle Zeit. Automatisiert agierende Systeme bieten hier eine Zeitersparnis und mehr Handlungsspielräume. Denkbar sind solche Systeme für eine Reihe an Naturkatastrophen. Erdbeben können über Sensorik frühzeitig erfasst werden, so dass Hochgeschwindigkeitszüge und Fahrstühle sofort abgebremst werden, ehe die sekundären, zerstörerischen Erdbebenwellen Wirkung entfalten. Hochwasser können durch Prognosen und Vorhersagen auf Basis weit entfernter Messpunkte vorhergesagt werden. Anhand von Satellitenbildern erkennen KIbasierte Algorithmen Vulkanausbrüche frühzeitig. ${ }^{25}$ In Japan werden Systeme zur Erkennung von Erdbeben, Tsunamis und Vulkanausbrüchen bereits eingesetzt. ${ }^{26}$ KI-Systeme bieten Möglichkeiten auf diese gerade wahrgenommenen (Sensor-) Daten wirklich zeitnah zu reagieren.

Ein besonderes Augenmerk der Verwaltung liegt auf den schutzbedürftigen Personen und gesellschaftlichen Gruppen, die sich oftmals nicht ausreichend selbst schützen können. Auch staatlich Bedienstete, vor allem im Außendienst, sind Gefahren physischer Gewalt ausgesetzt. Durch smarte Objekte generierte Sensordaten können in diesem Kontext auch zur Erkennung von Gefahrensituationen genutzt werden. Die Herausforderung besteht einerseits in der Erkennung der Gefahrensituation, in der Einleitung einer angemessenen Reaktion und andererseits in der angemessenen Berücksichtigung von Privatsphäre und Datenschutz. Künstliche Intelligenz ist bereits dazu in der Lage, aus gesprochener Sprache heraus Emotionen zu erkennen. Über die in Smartphones integrierte Lautstärkesensoren oder das Mikrophon kann die Gesprächsatmosphäre in Echtzeit analysiert werden, ohne wiederum die Gesprächsinhalte aufzuzeichnen oder zu analysieren. Wird eine der Situation unangemessene Emotion erkannt, so kann entweder der betreffenden Person eine Mitteilung ausgegeben oder direkt angemessene Maßnahmen eingeleitet werden.

Vgl. BAUER 2019.

26

Vgl. JaPAn Meterological AgEnCy 2016. 


\section{ANWENDUNGSFELDER FÜR KI IN DER JUSTIZ}

Auch im Kontext der Justiz finden sich zahlreiche Anwendungsmöglichkeiten für KI. Richter, Staatsanwälte, Rechtsanwälte und Justizbeamte können von Routineaufgaben entlastet und in ihren Entscheidungen unterstützt werden. Ebenso können bestimmte Entscheidungen auch vollständig automatisiert getroffen werden.

Die von der Justiz getroffenen Entscheidungen prägen Menschen und deren weitere Schicksale. Eine unabhängige und vertrauenswürdige Justiz stellt einen der Grundpfeiler einer freiheitlich demokratischen Grundordnung dar. Der Einsatz unterstützender oder entscheidener Systeme auf Basis von künstlicher Intelligenz muss daher maßvoll erfolgen, um das gesellschaftliche Vertrauen in die Arbeit der Justiz nicht dauerhaft zu beschädigen.

\section{Entscheidungsunterstützende Systeme in der Justiz}

Zahlreiche juristische Entscheidungen gehen mit einer Folge an Umsetzungen einher. Entscheidungen müssen nicht nur ordnungsgemäß und nachvollziehbar dokumentiert werden. Die Betroffenen müssen gemäß gesetzlicher Vorschriften auch über die Entscheidung sowie die daraus folgenden Rechte und Pflichten informiert werden. Zur Formulierung werden häufig standardisierte Textbausteine verwendet, welche entsprechend der Entscheidung eingefügt werden müssen. Unterstützende Systeme können den Bearbeiter hierbei entlasten, indem alle nötigen Dokumente im Nachgang einer Entscheidung automatisiert erstellt und den betreffenden Personen zugestellt werden. Oft werden Entscheidungen im Rahmen einer Anwendung von Rechtsentscheidungen auf den Einzelfall getroffen. Tagesgeld-Sätze sind entsprechend des Einkommens der jeweiligen Person zu berechnen. Verfahrenskosten sind auf den entsprechenden Streitwert anzupassen.

Daneben kann auch die juristische Entscheidung selbst durch den Einsatz technischer Systeme unterstützt werden. Künstliche Intelligenz bietet die Möglichkeit, Ähnlichkeiten einer Entscheidung zu bisherigen Fällen zu erkennen und dem Entscheider so Anhaltspunkte zu liefern. Dokumente und Aussagen können analysiert werden, inwiefern deren Aussagen übereinstimmen und wo sie sich widersprechen. Systeme können anhand des dargestellten Sachverhalts auch Empfehlungen geben, welche gesetzlichen 
Grundlagen in diesem Fall herangezogen werden sollten. All dies dient dazu, dem Entscheider eine möglichst fundierte Entscheidung treffen zu lassen. Er erhält Aufbereitungen, Kennzahlen und relevante Textstellen in aufbereiteter Form, sodass er die entsprechenden Inhalte in wesentlich kürzerer Zeit als bislang erfassen kann.

Auch seitens von Rechtsanwälten kann KI in gleicher Weise zur Unterstützung einer Verteidigung genutzt werden. Ebenso können Bürger automatisiert erste unverbindliche Einschätzungen der Erfolgsaussichten von Verfahren erhalten. Werden dem Anliegen offensichtlich keinerlei Chancen eingeräumt, kann der Bürger Zeit und Kosten sparen, während gleichzeitig die Justiz auch entlastet wird.

\section{Entscheidende Systeme in der Justiz}

Neben entscheidungsunterstützenden Systemen können in der Justiz auch Anwendungen genutzt werden, welche selbstständig Entscheidungen treffen. Bislang ist in Deutschland das Treffen von juristischen Entscheidungen dem Menschen vorbehalten. Urteile über Menschen werden immer von Menschen getroffen. Die Überlastung der Justiz in Deutschland lässt jedoch auch Überlegungen zur Automatisierung bestimmter Entscheidungen attraktiv erscheinen. Insbesondere sind es standardisierte Verfahren, welche in hohen Fallzahlen auftreten und die Kapazitäten der Justiz in hohem Maße in Anspruch nehmen.

Entscheidende Systeme in der Justiz bieten Potenziale in all jenen Bereichen, in denen fest definierte Verfahren mit klaren und messbaren Entscheidungskriterien existieren. Sind gewisse Voraussetzungen gegeben so wird eine zuvor festgelegte Entscheidung getroffen. Insbesondere eignen sich hierfür Verfahren, welche die Rechtsfolge an eindeutig messbare Kriterien binden. Solche Verfahren könnten vergleichsweise einfach ausschließlich durch technische Systeme bearbeitet werden, ohne dass ein Mensch in diesen Prozess mit involviert wird. Letztlich ist aber analog zu §31a SGB X sicherzustellen, dass alle im Verfahren relevanten Informationen auch tatsächlich berücksichtigt werden. Erst in einer möglichen zweiten Instanz würde dann das Ergebnis durch einen Menschen geprüft und gegebenenfalls revidiert werden können. 


\section{Mindestanforderungen}

Um tatsächliche Mehrwerte zu liefern und dauerhaft akzeptiert zu werden, haben unterstützende und entscheidende Systeme gewisse Anforderungen zu erfüllen. Sowohl Bürger als auch Angehörige der Justiz müssen mit den Systemen und deren Ergebnissen arbeiten und umgehen können. Ein Ziel ist es, Zeit und Ressourcen einzusparen. Transparente Bescheide, Widersprüche gegen Entscheidungen und das Anrecht auf die Bearbeitung eines Antrags durch einen Menschen müssen bestehen bleiben. Gleichzeitig besteht die Erwartung, dass durch den Einsatz dieser Systeme die Qualität der Entscheidungen deutlich verbessert werden kann.

Tonn und Stiefel ${ }^{27}$ zeigen als wichtigsten Punkt auf, dass im Angesicht immer komplexer Probleme durch Computer durchaus bessere Entscheidungen getroffen werden können. Durch die Einbindung verschiedener Akteure werde zudem die Schwarmintelligenz gefördert. Entscheidungen werden nicht mehr im Einzelfall durch einen einzelnen Entscheider getroffen. Stattdessen werden in die grundsätzliche, im System hinterlegte Entscheidung zahlreiche Experten einbezogen, sodass diese Entscheidung als wesentlich fundierter gelten kann. Sie sorgen weiterhin für einen transparenteren Entscheidungsprozess, wenn die Systeme den Vorgang transparent dokumentieren.

In einigen Fällen kann es jedoch unethisch sein, Entscheidungen an Maschinen abzugeben, da die Menschen dadurch ihre Menschlichkeit verlieren würden. Genauso werde die Fähigkeit leiden, Entscheidungen zu treffen. Menschen werden zudem weniger nach ethisch korrektem Handel streben. Die Autoren betonen außerdem, dass Computern Kreativität fehle und dass sie menschliche Werte nicht abbilden können. Ihnen fehle außerdem die Möglichkeit, abzuwägen oder sich einzufühlen. Schließlich müssen Menschen sich bei einer Entscheidungsübernahme durch Computer eingestehen, dass sie die schwereren Entscheidungen nicht treffen wollen - das sei unwahrscheinlich, so Tonn und Stiefel. ${ }^{28}$

Vgl. TonN/STIEFEL 2014, S. 134 ff.

28

Vgl. TonN/StiEfEl 2014, S. 134 ff. 
Eine grundlegende Frage betrifft zudem die Anforderungen an die Qualität der verwendeten Daten. Mehr Daten sind nicht unbedingt gute Daten. Gefälschte Fakten und unzureichende Informationen können zu anderen Urteilen führen. Qualität und Richtigkeit müssen sichergestellt werden. Unter diesem Punkt debattiert Novoselic ${ }^{29}$ auch den Punkt des Ermessens. Dieser hänge außerdem damit zusammen, dass Politik oft Ursachenforschung erfordere, die bei einer reinen Dateninterpretation zu kurz komme. Der Autor spricht außerdem die Gefahr mangelnden Datenschutzes und zunehmender Überwachung an, wenn Sensoren, smarte Objekte und cyberphysischen Systeme laufend Daten generieren, die sich durch KI auswerten lassen. Weiterhin ergebe sich das Problem der Verantwortlichkeit und Rechenschaft. Wer verantwortet von Systemen getroffene Entscheidungen? Schließlich spricht Novoselic die Gefahr an, dass sich neue elitäre Gruppen bilden, die als einzige die Technologie verstehen und somit politische Prozesse beeinflussen. Gleichzeitig seien Daten nie neutral, ihre Auswahl werde immer auch von den zuständigen Experten beeinflusst. Es entstehe aber eine Illusion von Objektivität.

Kennedy und Scholl ${ }^{30}$ betonen einen ähnlichen Punkt. Alle Modelle haben immer eine implizite Beeinflussung durch die Experten, von denen sie erstellt werden. Die Ergebnisse verleiten zu einem Objektivitätsglauben, der nicht angemessen sei. Dieser werde durch Informationstechnologie noch verstärkt. Genauso betonen sie die Bedeutung menschlichen Ermessens und Wertevorstellungen. Die Autoren sehen außerdem die Gefahr, dass es nicht gelingen könne, wichtige Grundsätze der Gleichheit vor dem Gesetz in Informationssysteme zu übersetzen. Sie sehen außerdem die Unabhängigkeit und die Privatheit gefährdet. Es bestehe die Gefahr der Manipulation. Es müsse zudem sichergestellt werden, dass es möglich sei, mit Fehlentscheidungen umzugehen, die auch ein Computersystem begehe. Sie sehen außerdem statt wachsender eher abnehmende Transparenz von Entscheidungsprozessen, besonders wenn die Entscheidungen von Systemen getroffen werden, deren Code nicht öffentlich sei. Zudem wird die Schwierigkeit beschrieben, von juristischer Logik in Computerprogrammierung zu übersetzen, besonders, weil es kaum Experten mit Brillianz in beiden Feldern gebe.

Vgl. Novoselic 2016, S. $134 \mathrm{ff}$. 
Schließlich fragen sich die Autoren, wie mit nicht vorhergesehenen Eingaben, Informationen oder anderer notwendiger Flexibilität umgegangen werden könne. ${ }^{31}$

\section{E. NEUE FORSCHUNGSSCHWERPUNKTE}

Aus den beschriebenen Debatten ergibt sich eine eindrucksvolle Liste von Fragen, die die Verwaltungsinformatik, die Rechtsinformatik und die Politikinformatik zusammen mit ihren anderen Schwesterdisziplinen (Verwaltungswissenschaft, Politikwissenschaft, Rechtswissenschaft, Psychologie, Informatik) in den nächsten Jahren zur nachhaltigen wie fairen Nutzung von künstlicher Intelligenz im öffentlichen Sektor interdisziplinär bearbeiten müssen. ${ }^{32}$

Diese betreffen zunächst die als Entscheidungsgrundlagen fungierenden Daten. Die Qualität von Entscheidungen kann immer maximal der Qualität der zugrundeliegenden Daten entsprechen. Aus diesem Grund stellt sich die Frage, wie gut und wertvoll Daten vorliegen, die durch künstliche Intelligenz und autonome Systeme ausgewertet werden können. Nicht nur klassische staatliche Datenbestände bieten hierfür bereits eine breite Grundlage. Durch smarte Objekte generierte Daten werden in Zukunft in völlig neuen Dimensionen Informationen liefern, die in Entscheidungen einbezogen werden können. Hierbei stellt sich jedoch die Frage, inwieweit alle entscheidungsrelevanten Informationen durch Daten abgebildet werden können und an welchen Stellen stattdessen eine subjektive, menschliche Einschätzung des Entscheiders notwendig ist. Während bestimmte Verfahren auf eindeutig messbaren und quantifizierbaren Werten und Kennzahlen basieren, verlangen andere eine individuelle Einschätzung. Die geeigneten Verfahren sind daher bereits im Hinblick auf die zur Entscheidung notwendigen Informationen in einem ersten Schritt zu identifizieren. Ebenso muss aber auch die Sicherung der Datenqualität miteinbezogen werden. Insbesondere die Nutzung smarter Daten stellt ein neues Feld dar. Daten können sowohl für ein faktenbasiertes Handeln genutzt, aber auch gezielt manipuliert werden, um Entscheidungen zu beeinflussen.

Vgl. vON LUCKE/GROßE 2017, S. 322-324.

32

Vgl. vON LUCKE/GROßE 2017, S. 324-327. 
Dies muss bei der Gestaltung Berücksichtigung finden. Daher ist im Vorfeld festzulegen, welche Daten aus welchen Quellen in Entscheidungsprozessen genutzt werden können und welchen Vertrauensniveaus diese entsprechen müssen. Werden diese Anforderungen nicht erfüllt, ist von einem Einsatz abzusehen.

Gleichzeitig ist auch der Entscheidungsprozess im engeren Sinne zu betrachten. Dabei stellt sich die grundlegende Frage, welche Entscheidungsarten es gibt und inwieweit sich diese zur Automatisierung oder Unterstützung von Entscheidungen eignen. Wie oben bereits erwähnt scheinen sich gebundene Entscheidungen besser zu eignen, da die Rechtsfolge bei gegebenem Sachverhalt bereits durch den Gesetzgeber zwingend vorgegeben ist. Dabei stellt sich die konkrete Frage, wie Prozesse bei Fragestellungen ohne, aber zukünftig auch mit Ermessensspielraum zu gestalten sind. Damit geht auch die grundlegende Frage einher, welche Rolle Beweggründe, Einzelschicksale und Ursachenforschung spielen sollen. Reicht es, wenn definierte Fakten vorliegen, oder sollen auch die dahinter liegenden Informationen berücksichtigt werden? Wie soll mit unvorhergesehenen Informationen umgegangenen werden? Ebenso müssen auch auf künstlicher Intelligenz basierende Systeme Grundsätze des Rechtsstaats wie die Chancengleichheit der Akteure beachten. In der Umsetzung stellt sich zudem die Frage, wie die juristische Entscheidungslogik in die Programmierung technischer KI-Systeme übersetzt werden kann. Eine elementare Rolle wird auch die Transparenz und Nachvollziehbarkeit von algorithmischen Entscheidungen spielen. Vertrauen und Akzeptanz in KISysteme kann nur geschaffen werden, indem die verwendeten Informationen und die Logik der Entscheidung für den Menschen nachvollziehbar dargelegt werden.

Fragen stellen sich auch hinsichtlich der Umsetzung von Entscheidungen autonomer Systeme. Wie wird mit autonom getroffenen Entscheidungen innerhalb und außerhalb von Organisationen umgegangen? Verringern sich durch automatisierte Entscheidungen Konfliktpotenziale? Ist das Vertrauen in Entscheidungen größer, wenn diese von KI-basierten Systemen getroffen werden? Wie kann für breite Akzeptanz von KI-basierten Entscheidungen gesorgt werden?

Nicht zuletzt stellen sich aber auch Fragen nach den Rahmenbedingungen und der Umwelt autonom entscheidender Systeme. Wie verändert sich die generelle Nachvollziehbarkeit von Entscheidungen, wenn autonome Systeme anstatt von Menschen Entschei- 
dungen treffen? Welche Entscheidungen sollten aus ethischen oder moralischen Gründen auch weiterhin ausschließlich durch Menschen getroffen werden? Wie sollte mit Verantwortlichkeit und Rechenschaft umgegangen werden? Wie kann verhindert werden, dass nur noch wenige Experten Entscheidungen nachvollziehen und kontrollieren können? Wie wirkt sich die Abgabe von Entscheidungen auf die Fähigkeiten künftiger Generationen aus? Und nicht zuletzt, welche Abhängigkeiten von technischen Systemen entstehen und wie können entscheidende Systeme auch wieder abgestellt werden?

\section{F. $\quad$ FAZIT}

Staat, Verwaltung und Justiz müssen sich in Zeiten von Smart Government und künstlicher Intelligenz auf substanzielle Veränderungen durch die neuen Möglichkeiten des Internets der Dinge und des Internets der Dienste einstellen.

Die Einführung selbstentscheidender autonomer Systeme in Staat und Verwaltung ist nicht trivial. Sie darf auch nicht auf die leichte Schulter genommen werden, denn sie hat grundlegende Folgewirkungen für Staat, Verwaltung und Gesellschaft. Positive und negative Konsequenzen lassen sich nicht immer in ihrem vollen Umfang vorhersehen. Rund um vier Herausforderungen sind in diesem Beitrag zahlreiche aktuelle wie offene Fragestellungen zusammengetragen worden. Für eine erfolgreiche Umsetzung autonomer Systeme sind Antworten erforderlich, wie verlässliche Entscheidungsgrundlagen geschaffen, wie Entscheidungen von autonomen Systemen getroffen werden, wie die Umsetzungen von Entscheidungen autonomer Systeme realisiert wird sowie welche Rahmenbedingungen erforderlich sind. Diese Herausforderungen skizzieren eine Forschungsagenda, die die Verwaltungsinformatik, Rechtsinformatik und die Politikinformatik zusammen mit der Verwaltungswissenschaft, der Politikwissenschaft, der Rechtswissenschaft, der Psychologie, der Wirtschaftsinformatik und der Informatik in den kommenden Jahren inter- und transdisziplinär zu bearbeiten haben. Staat und öffentliche Verwaltung wären gut beraten, sich mit der Wissenschaft diesen Fragestellungen zeitnah zu stellen. Sie wären dann frühzeitig in der Lage, Antworten und Rahmenempfehlungen geben zu können, wenn die Umsetzung von Smart Government und autonomen Systemen in den kommenden Jahren ansteht. In diesem Zusammenhang 
muss aber ebenso über Grenzen, Ethik und eine Regulierung des Einsatzes von künstlicher Intelligenz nachgedacht, offen debattiert und politisch gestritten werden. Eine solche Transformation kann nicht nur zu Mehrwerten und Gewinnern führen, sondern wird auch Verlierer und Verluste hervorbringen. Der Einsatz von KI durch autoritäre Polizeistaaten zur Erfassung, Verfolgung und Inhaftierung andersdenkender Staatsbürger weist ebenso menschenverachtende Züge auf wie der Einsatz autonomer Waffensysteme in militärischen Konflikten. Handlungsbedarf ist gegeben. Vorhandene Gestaltungsspielräume gilt es gemeinsam zu nutzen. Die interdisziplinär aufgestellten Wissenschaften werden dabei wertvolle Partner sein müssen.

\section{LITERATURVERZEICHNIS}

AreEKADAN, J. 2018: Alexa nennt Wartezeiten, in: Kommune 21, 18 (2), S. 22-23.

BAUER, V. 2019: Wie KI und Satelliten helfen können, Vulkanausbrüche vorherzusagen, Mobile Geeks, 09.03.2019. Online: https://www.mobilegeeks.de/news/wie-ki-und-satelliten-helfen-koennen-vulkanausbrueche-vorherzusagen/.

BRAUN BINDER, N. 2016b: Vollständig automatisierter Erlass eines Verwaltungsaktes und Bekanntgabe über Behördenportale. Die öffentliche Verwaltung, S. 891-898.

CUmmingS, D. 2016: Seven Spectrum of Outcomes for AI, in: David Cummings on Startup, Atlanta. Online: https://davidcummings.org/2016/12/28/seven-spectrum-of-outcomes-for-ai.

BERGMANN, L.; CRESPO, I.; FLEISCHMANN, J. 2009: Gestaltung transparenter Geschäftsprozesse. In: Dombrowski, U.; Hermann, C.; Lacker, T.: Sonnentag, S.: Modernisierung kleiner und mittlerer Unternehmen, Springer, Heidelberg.

DAUM, R. 2002: Integration von Informations- und Kommunikationstechnologien für bürgerorientierte Kommunalverwaltungen, Nomos Verlagsgesellschaft, Baden-Baden.

DJEFFAL, C. 2017: Künstliche Intelligenz in der öffentlichen Verwaltung, Berichte des NEGZ, Berlin. Online: https://www.hiig.de/wp-content/uploads/2019/o3/NEGZ-Kurzstudie-3-KuenstlIntelligenz-20181113-digital.pdf. 
DUNCKER, K.; NOLTEMEIER, A. 1985: Organisationsmodelle für ein Bürgeramt und deren Realisierung in der Stadt Unna, Verlag, St. Augustin und Darmstadt.

ETSCHEID, J. \& VON LUCKE, J. 2020: Künstliche Intelligenz in der öffentlichen Verwaltung, Digitalakademie@bw, Stuttgart, in Druck.

GOLDACKER, G. 2017: Die Perspektive wechseln, Kommune 21, 07.03.2017. Online: https://www.kommune21.de/meldung_2590o_Die+Perspektive+wechseln.html.

GrunOw, D. 1988: Bürgernahe Verwaltung - Theorie, Empirie, Praxismodelle, Campus Verlag, Frankfurt und New York.

Japan Meteorological Agency 2016: The national meteorological service of Japan, Tokio. Online: http://www.jma.go.jp/jma/en/Activities/brochure201603.pdf.

KenNEDY, R. \& SCHOLL, H. J. 2016: E-regulation and the rule of law: Smart government, institutional information infrastructures, and fundamental values, Information Polity, 21(1), S. 77-98. http://doi.org/10.3233/IP-150368.

KNOBLOCH, T. 2018: Vor die Lage kommen: Predictive Policing in Deutschland, Stiftung Neue Verantwortung, Berlin.

KÖNEKE, V. 2018: Doktor Algorithmus, sag mir was ich hab, Zeit Online, 13.08.2018. Online: https://www.zeit.de/digital/internet/2018-o8/deep-learning-medizin-kuenstliche-intelligenz-neurologie-augenheilkunde.

KuHN, J. 2019: Mein Smartphone weiß dass ich wütend bin, in: Süddeutsche Zeitung, 27.03.2019. Online: https://www.sueddeutsche.de/digital/smartphone-software-emotionen-simulation-ki-1.4377004.

LANZ, A. 2010: Entwurf und Implementierung eines Prozesses aus der Verwaltung am Beispiel einer Reisekostenabrechnung, Universität Ulm, Ulm.

LINDINGER, M. 2019: KI entdeckt verborgenes Wissen, in: Frankfurter Allgemeine Zeitung, 30.07.2019. Online: https://www.faz.net/aktuell/wissen/klug-verdrahtet/klugverdrahtet-ki-entdeckt-beim-lesen-von-artikeln-verborgenes-wissen-16286851.html.

VON LUCKE, J. 2008: Hochleistungsportale für die öffentliche Verwaltung, Eul-Verlag, Siegburg. 
VON LUCKE, J. 2016: Smart Government - Intelligent vernetztes Regierungs- und Verwaltungshandeln in Zeiten des Internets der Dinge und des Internets der Dienste, Schriftenreihe des The Open Government Institute | TOGI der Zeppelin Universität Friedrichshafen, Band 16, Berlin: epubli GmbH.

VON LUCKE, J. 2018a: In welcher smarten Welt wollen wir eigentlich leben? Verwaltung und Management, 24(4), S. 177-196.

VON LUCKE, J. 2018b: Smart Government auf einem schmalen Grat, in: Resa Mohabbat Kar, Basanta Thapa, Peter Parycek (Hrsg.): (Un)Berechenbar? Algorithmen und Automatisierung in Staat und Gesellschaft, Kompetenzzentrum Öffentliche IT (ÖFIT) Fraunhofer-Institut für Offene Kommunikationssysteme FOKUS, Berlin.

VON LUCKE, J. \& GROßE, K. 2017: Smart Government - Offene Fragen zu autonomen Systemen im Staat 4.o, in: Schröter, W. (Hrsg.): Autonomie des Menschen - Autonomie der Systeme - Humanisierungspotenziale und Grenzen moderner Technologien, Talheimer Sammlung kritisches Wissen, Band 71, Talheimer Verlag, Mössingen-Talheim 2017, S. 313-327.

MAINZER, K. 2016: Künstliche Intelligenz - Wann übernehmen die Maschinen? Springer Verlag, Heidelberg.

MÖltGen, K. \& LORIG, W. 2009: Die kundenorientierte Verwaltung - zu den Facetten eines Leitbildes der Verwaltungsmodernisierung, VS Verlag für Sozialwissenschaften, Wiesbaden.

NeSSELDREHER, A. 2006: Entscheiden im Informationszeitalter, Der Andere Verlag, Tönning.

Novoselic, S. 2016: Smart Politics, in: von Lucke, J. (Hrsg.): Smart Government -Intelligent vernetztes Regierungs - und Verwaltungshandeln in Zeiten des Internets der Dinge und des Internets der Dienste, TOGI Schriftenreihe (16), epubli, Berlin, S. 77-95. PÜTTNER, G. 2000: Verwaltungslehre - Ein Studienbuch, 3. Auflage, Verlag C.H. Beck, München. 
SCHEER, A.-W. 2017: Robotic Procress Automation (RPA) - Revolution der Unternehmenssoftware, IM+io - Das Magazin für Innovation, Organisation und Management, 32 (3), S. 30-41.

SIEGEL, T. 2017: Automatisierung des Verwaltungsverfahrens - zugleich eine Anmerkung zu §§ 35a, 24 I 3, 41 IIa VwVfG, in: Deutsches Verwaltungsblatt (132), S. 24-28.

StANOEVSKA-SLABEVA, K. 2018: Conversational Interfaces - die Benutzerschnittstelle der Zukunft?, in: Wirtschaftsinformatik und Management, 10 (6), S. 26-37.

Stucki, T.: D'OnofRio, S. \& Portmann, E. 2018: Chatbot - Der digitale Helfer im Unternehmen. HMD Praxis der Wirtschaftsinformatik, 55, S. 725-747.

TONN, B. \& STIEFEL, D. 2014: Human Extinction Risk and Uncertainty: Assessing Conditions for Action. Futures 63:134-44.

WANG, R. 2016: Monday's Musings - Understand The Spectrum Of Seven Artificial Intelligence Outcomes, Constellation Research. Online: http://blog.softwareinsider.org/2016/o9/18/mondays-musings-understand-spectrum-seven-artificial-intelligence-outcomes/.

Welzel, C. \& Grosch, D. 2018: Das ÖFIT-Trendsonar künstliche Intelligenz, Kompetenzzentrum öffentliche IT, Berlin. 